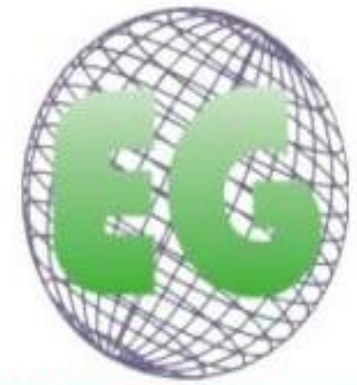

ISSN 1695-6141 $N^{\circ} 45$

\title{
Programas educativos en pacientes con Enfermedad Pulmonar Obstructiva Crónica. Revisión integradora
}

Educational programs for patients with Chronic Obstructive Pulmonary Disease. Integrative Review

\section{${ }^{*}$ Folch, Ana ${ }^{* *}$ Orts-Cortés, Maria Isabel ${ }^{* * *}$ Hernández-Carcereny, Carmen ***Seijas-Babot, Nuria **Maciá-Soler, Loreto}

*Universitat Jaume I. Castellón de la Plana. E-maill: afolch@uji.es **Universitat de Alicante.

***Hospital Clinic. Barcelona. España.

http://dx.doi.org/10.6018/eglobal.16.1.249621

\section{RESUMEN}

Según la OMS, el objetivo de la educación terapéutica (ET) es ayudar al paciente a adquirir los recursos necesarios para gestionar óptimamente su vida con una enfermedad crónica. La ET forma parte del tratamiento en la prevención secundaria y terciaria. La última revisión de la Cochrane ha demostrado resultados positivos, sin embargo, la heterogeneidad de las poblaciones, tipo de intervenciones y medidas de resultado hace que sea difícil establecer recomendaciones claras en este ámbito. Así como se desconoce qué instrumentos son los recomendables para evaluar el cumplimiento terapéutico en la EPOC. Por tanto es necesario realizar de una revisión con el objetivo de conocer las características de los ensayos clínicos, el perfil del paciente EPOC, los contenidos de la ET y las variables estudiadas en los mismos. Identificándose 22 ensayos clínicos con una alta calidad metodológica, escala Jadad $>3$ que mostraron que en los ensayos clínicos de programas de ET las variables más estudiadas son la calidad de vida, ingresos hospitalarios y las variables de función pulmonar, donde la ET disminuye los ingresos hospitalarios, favorece la deshabituación tabáquica, mejora los conocimientos de la EPOC cuyas intervenciones son sesiones grupales, con diferentes materiales educativos, existiendo un auge en el número de publicaciones en este ámbito. Existe una gran variedad en los instrumentos de evaluación y heterogeneidad de las intervenciones, para establecer conclusiones.

Palabras clave: EPOC; educación en salud

\section{ABSTRACT}

According to $\mathrm{WHO}$, the aim of therapeutic education (TE) is help the patient to acquire the resources necessary to optimally manage your life with a chronic disease. The TE is part of treatment in secondary and tertiary prevention. The latest Cochrane review has shown positive results however, 
heterogeneity of populations, types of interventions and outcome measures makes it difficult to establish clear recommendations in this area. And what instruments are recommended to assess the therapeutic education in patients with COPD. It is therefore necessary a review with the aim of knowing the characteristics of clinical trials, the profile of COPD patient, the contents of ET and the variables studied in them. In this review are Identified 22 clinical trials with high methodological quality, Jadad scale> 3. Which showed that in clinical trials programs ET the most studied variables are the quality of life, hospital admissions and lung function variables, where ET decreases hospital admissions, promotes smoking cessation and improving knowledge of COPD. Whose interventions are group sessions with different educational materials. There being a rise in the number of publications in this area, there is a great variety of assessment tools and heterogeneity of interventions, to draw conclusions.

Keywords: COPD; nursing health education

\section{INTRODUCCIÓN}

La enfermedad pulmonar obstructiva crónica (EPOC) se caracteriza por la presencia de una limitación crónica y poco reversible del flujo aéreo, asociada a una reacción inflamatoria anómala, debida principalmente al humo del tabaco ${ }^{(1,2)}$.

En la EPOC aparecen periodos de empeoramiento brusco de los síntomas, conocidos como exacerbaciones 0 agudizaciones ${ }^{(3)}$, que se definen como episodios de inestabilidad clínica que acontecen en el curso natural de la enfermedad y se caracterizan por un empeoramiento mantenido de los síntomas respiratorios que van más allá de sus variaciones diarias ${ }^{(4)}$. Estas agudizaciones ocupan un lugar destacado en el trascurso de la enfermedad ${ }^{(5)}$, con un promedio de 1 a 4 agudizaciones anuales ${ }^{(6)}$, afectando notablemente a la calidad de vida relacionada con la salud (CVRS) ${ }^{(7)}$ por una discapacidad progresiva en lugar de una muerte inmediata $^{(8)}$.

Este acontecimiento genera una fuerte carga asistencial y económica. En el año 2012 en España, 38.726 personas enfermas de EPOC, ocasionaron 664.545 ingresos hospitalarios con una estancia media de 8 días de ingreso ${ }^{(9)}$. En 2020 se espera que sea la tercera causa de muerte en el mundo ${ }^{(10)}$. Exacerbaciones también acrecentadas por la presencia de comorbilidades asociadas, como asma, problemas cardíacos o hipertensión ${ }^{(11)}$. Parece necesario estudiar opciones de prevención de las exacerbaciones que padecen las personas con EPOC.

La educación representa hoy una práctica indisociable en la terapéutica de este tipo de pacientes, como así queda reflejado en la Estrategia Nacional del Paciente con $\mathrm{EPOC}^{(3)}$, donde se plantea la educación terapéutica como un método para la reducción de la frecuencia de las agudizaciones, mejora de la CVRS, la disnea medida mediante el Medical Result Council (MRC).

Entendiendo según la OMS que la educación terapéutica es un proceso continuo, integrado en los cuidados y centrado sobre el paciente. Que comprende actividades organizadas de sensibilización, información, aprendizaje y acompañamiento psicosocial relacionado con la enfermedad y el tratamiento prescrito. Contempla ayudar al paciente y a sus educadores, vivir lo más sanamente posible y mantener o mejorar la calidad de vida y así poder gestionar óptimamente su vida con la enfermedad ${ }^{(12)}$.

Dos de las últimas revisiones Cochrane ${ }^{(8,13)}$ publicadas, ponen de manifiesto la mayor capacidad de los pacientes con EPOC en reconocer y reaccionar de manera adecuada ante una exacerbación después de haber recibido contenidos de educación 
terapéutica, existiendo controversia en los contenidos por la menor o mayor eficacia de los mismos.

Por tanto, el objetivo principal del presente estudio es realizar una revisión integradora de la literatura, para identificar estudios que evaluaran intervenciones educativas en pacientes con EPOC.

Los objetivos secundarios fueron:

1. Conocer las características sociodemográficas.

2. Conocer las variables resultado y los instrumentos de valoración.

3. Conocer la calidad metodológica de los ensayos clínicos estudiados.

4. Conocer los contenidos de las intervenciones de educación terapéutica

\section{METODOLOGÍA}

\section{Diseño}

Revisión integradora de la literatura científica sobre estudios de intervención basados en programas de educación terapéutica en pacientes con EPOC, en la base internacional de Medline (Pubmed) y búsqueda en todas las referencias de los artículos incluidos.

\section{Criterios de selección}

\section{$\underline{\text { Tipo de estudio }}$}

Ensayos clínicos controlados, aleatorizados o no aleatorizados que evaluaban la implantación de un programa de educación terapéutica en pacientes con un diagnóstico clínico de EPOC sin tener en cuenta el año de realización ni el idioma. Criterios de exclusión, estudios con una puntuación entre 0 y 2 puntos en la escala Jadad.

\section{$\underline{\text { Tipos de participantes }}$}

Pacientes con EPOC primario, diagnosticados por un profesional de la salud, mediante criterios espirométricos (FEV1<80\% del valor teórico y un FEV1/CVF $<70 \%$ del valor teórico). Se excluyeron los estudios de participantes con diagnóstico primario de asma.

\section{$\underline{\text { Tipo de intervención }}$}

Las intervenciones se clasificaron, en base a si los estudios incluían o no educación sobre la EPOC. La definición educación sobre EPOC utilizada fue: programa que transfiere información sobre la EPOC y su tratamiento en cualquiera de las siguientes formas: escrita, verbal, visual o auditiva. La educación mínima incluyó la provisión de material escrito o una breve interacción verbal estructurada entre un profesional de asistencia sanitaria y un paciente. Sin embargo, tenían que enmarcarse en un programa formal, en el que el primer objetivo era mejorar el conocimiento y la comprensión de la EPOC. El programa educativo podía dirigirse al abandono del hábito tabáquico, instrucción en la técnica de la inhalación, mejora de sus hábitos de vida, autocuidado durante las exacerbaciones, capacidad para realizar las actividades de la vida diarias o una combinación de las anteriores. 
Cualquiera de los siguientes resultados: CVRS, variables funcionales, FEV (Volume exhaled during the first second of a forced expiratory), saturación de oxígeno, walking test, disnea Medical Resoult Council (MRC), Staging System for Chronic Obstructive Lung Disease (GOLD), comorbilidades, signos y síntomas, adherencia farmacológica, consumo de tabaco, consumo de alcohol, medicación utilizada, conocimiento de la EPOC, uso de recursos sanitarios (ingresos hospitalarios, visitas a urgencias, número de exacerbaciones), índice de masa corporal (IMC), ansiedad, depresión y satisfacción personal.

\section{Identificación de los estudios}

Para la búsqueda se utilizaron los descriptores del Medical Subject Headings (MeSH), "COPD " y "education" con el operador booleano "and" entre ambos. También se realizó una búsqueda de todas las referencias bibliográficas de los artículos incluidos. No se incluyen en la revisión los resultados no publicados. No hubo restricciones con respecto al lugar de la investigación, idioma o fechas de publicación, es decir, desde el comienzo de indexación de cada base, hasta marzo de 2014.

\section{Valoración de la calidad metodológica}

La evaluación de la calidad metodológica, fue realizada por pares mediante la utilización de la escala Jadad, que evalúa la calidad de los estudios incluidos, utilizando un sistema de calificación en base a las respuestas de las 7 preguntas que plantea el cuestionario. Cada pregunta afirmativa se suma 1 punto y cada pregunta negativa 0 puntos, las 2 preguntas funcionan de manera distinta si su respuestas son afirmativas se suma -1 y en el caso de negación se sumaría 0 puntos. Considerándose estudios de baja calidad entre 0-2 puntos y de buena calidad entre 35 puntos. Los ítems evaluados son la asignación al azar, el cegamiento y la descripción de las perdidas y motivos de abandono ${ }^{(14)}$. Seleccionando a todos los estudios que tenían una valoración de la calidad metodológica entre 3 y 5 puntos, siendo excluidos los artículos con una puntuación entre 0 y 2 puntos en la escala Jadad. El cegamiento de los participantes no fue penalizado debido a la naturaleza de la intervención de los ensayos clínicos.

\section{Análisis de los datos}

Se realizó un análisis cualitativo de los datos obtenidos a partir de la plantilla previamente elaborada para tal fin. De los datos obtenidos se ha realizado un análisis exhaustivo del diseño del estudio, edad media, sexo predominante, tipo de centro de realización del estudio (universidad, hospitalario, atención primaria), número de participantes, número de pacientes asignados al grupo control, número de pacientes asignados al grupo intervención, programa de intervención realizado en cada estudio, variables medidas, periodo de seguimiento, evaluación y resultados obtenidos. Así como se realizó un análisis descriptivo de las variables, categorizándolas como efecto favorable, igual o desfavorable. Ya que en diversos estudios las variables han sido analizadas mediantes escalas de valoración distintas, aspecto también puesto de manifiesto en otra revisión ${ }^{(8)}$. 


\section{RESULTADOS}

Se identificaron 321 artículos con la estrategia antes citada. Tras la lectura del título y resumen se seleccionaron 58 artículos que podían cumplir con los criterios de selección. A continuación se realizó una lectura completa del artículo para verificar los criterios de selección, quedando un total de 28 artículos seleccionados. Los 30 artículos desechados fueron excluidos por los siguientes motivos:

Protocolos de investigación sin resultados $(n=7){ }^{(15-21)}$ incluían a pacientes con EPOC y pacientes sanos $(n=3)^{(22-24)}$ los pacientes asignados al grupo control no tenían una atención convencional $(n=7){ }_{(25-31)}^{(37)}$ estudio repetido $(n=5)^{(32-36)}$ la intervención era un programa de canto $(n=1){ }^{(37)}$, no tenía una intervención educativa $(n=2)^{(38,39)}$ se centraba principalmente en un programa de rehabilitación pulmonar basado en ejercicios físicos $(n=3)^{(40-42)}$, se basaba en un abstract de un congreso $(n=1)^{(43)}$ u otros cuyo objetivo era validar un cuestionario para la tos $(n=1)^{(44)}$. Se realizó una búsqueda manual de las referencias de los 28 artículos que cumplían los criterios de selección ${ }^{(5,45-71)}$ con la obtención de 4 artículos ${ }^{(72-75)}$ más. Con la obtención de 32 artículos, que fueron valorados por su calidad metodológica, excluyendo a $(51,52,56,59,61,65,66,68,72)$ por puntuaciones en la escala de Jadad entre $0-2$ puntos. Finalmente $22 \quad(5,45-50,53-55,57,58,60,62-64,67,69-71,73-75)$ artículos fueron incluidos y clasificados según: diseño, país, tipo de centro, participantes totales, participantes asignados al grupo control (GC), participantes asignados al grupo intervención (Gl), intervención educativa, variables cuantificadas, periodo de seguimiento y resultados obtenidos.

\section{Participantes/ reclutamiento}

En el total de artículos encontrados se asignaron al azar un total de 8369 pacientes; $7211(76,2 \%)$ pacientes completaron estos estudios. Las tasas de abandono oscilaron entre el $0 \%$ y $66,3 \%$, con una media de $20,8 \%$ en número medio de abandonos. De los 22 estudios, $12(54,6 \%)$ estudios reclutaron a sus pacientes en hospitales, 8 $(36,4 \%)$ estudios en centros de atención primaria, $1(4,6 \%)$ en un centro universitario y en otro estudio incluido no indicaba el tipo de centro ${ }^{(53)}$.

En el $45,5 \%(n=10)$ de los estudios fueron mayoría los hombres, frente a un 40,9\% $(n=9)$ donde la mayor prevalencia eran mujeres y en un $13,6 \%(n=3)$ fue equitativo el reparto entre sexos, todos los estudios llevados a cabo en España muestra unas diferencias muy significativas de la prevalencia de la EPOC en el sexo masculino. La media de edad de todos los estudios es de 66,4 años, siendo la comorbilidad más frecuente las enfermedades coronarias, de los estudios que así lo analizaron $(n=5)^{(45,46,60,67,73)}$.

En uno de los estudios que así lo recogen afirman que el $90 \%$ de los pacientes se vacunó contra la gripe el año anterior, no teniendo los resultados de incidencia de la vacunación tras la implementación del programa de educación terapéutica ${ }^{(47)}$.

La mitad de los estudios desarrolló su estudio con un periodo de seguimiento de un año de duración $(n=10)$, siguiéndole los estudios que han tenido menos de un año de duración $(n=9)$, los que han tenido una duración de dos años $(n=2)$ así como un estudio no reveló la duración del mismo ${ }^{(74)}$. Se observó una gran heterogeneidad en los periodos de evaluación durante el trascurso de las mismas, realizándose con mayor prevalencia de dos evaluaciones, estudios de antes y después. 
Diagrama 1. Diagrama de flujo del estudio que muestra la metodología del trabajo

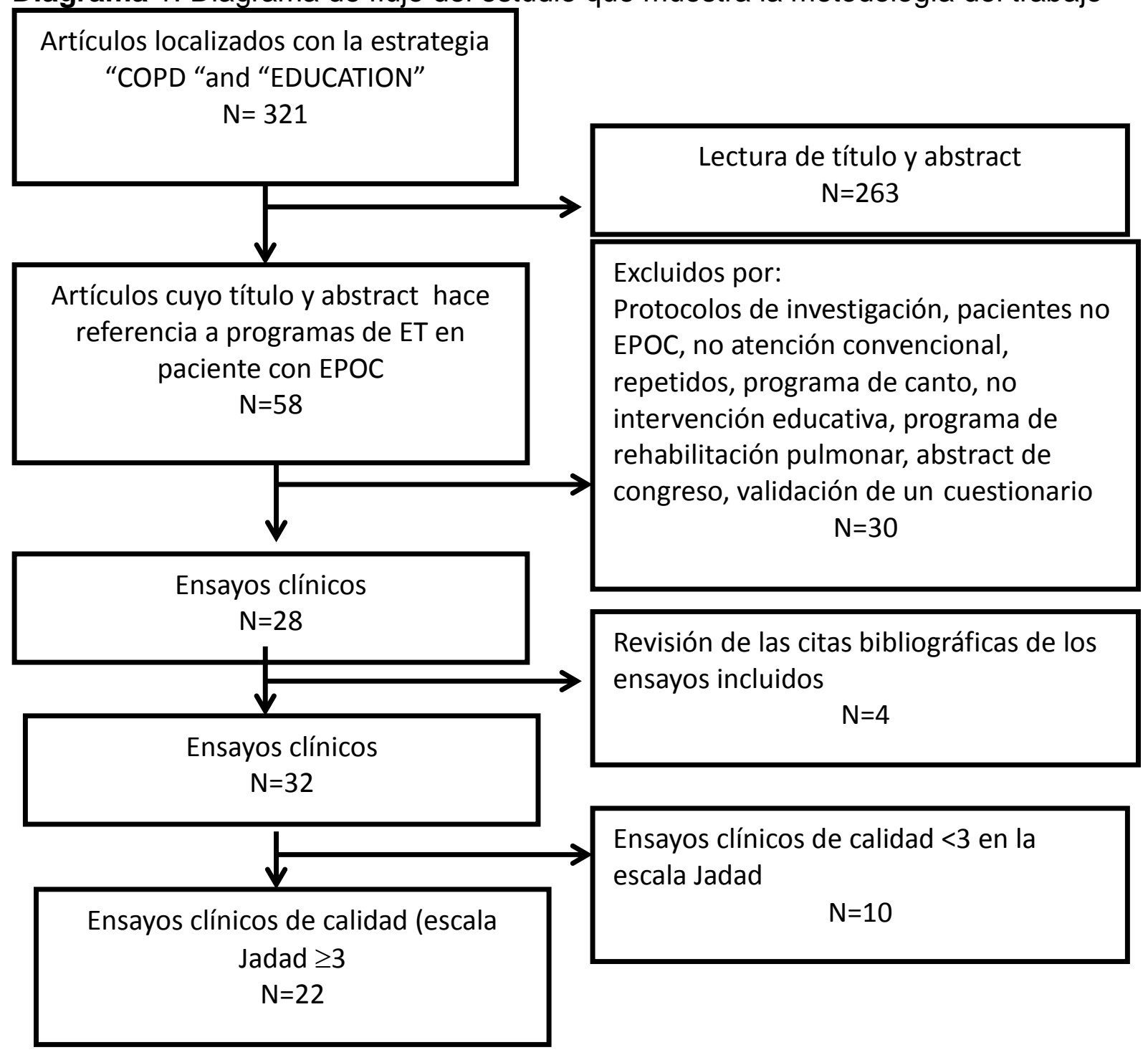

\section{Intervenciones}

Los 22 estudios describieron la educación en autocuidados en la EPOC comparada con la atención convencional. En todos los estudios, salvo uno ${ }^{(64)}$, el modo de educación fue grupal, en algunos de los casos además se realizó de un plan individual $(n=9)(45,46,48,55,60,64,71,73,74)$, uso de folletos informativos para reforzar la educación $(n=8)$ $(5,45,48,53,54,60,67,71)$, seguimiento telefónico $(n=3)^{(45,70,71)}$ o utilización de diario $(n=3)$ $(50,53,71)$. El contenido de las sesiones educativas fue: en 18 artículos $(81,8 \%)$ en sus sesiones educativas hablan sobre el conocimientos sobre la EPOC, con los siguientes componentes: anatomía y fisiología $(n=3)^{(50,54,63)}$, descripción de la enfermedad $(n=10)^{(5,45-47,49,55,60,64,67,70)}$, signos y síntomas de la EPOC $(n=3)^{(54,57,74)}$, signos y síntomas de las exacerbaciones $(n=10)(50,54,55,57,60,63,64,67,70,74)$, diagnóstico de la EPOC $(n=3)^{(57,60,63)}$, desencadenantes de una exacerbación $(n=6)^{(50,60,63,64,67,70)}$ y servicios hospitalarios de los cuales puede hacer uso $(n=3)^{(47,67,74)}$.

Otro de los apartados fue el tratamiento no farmacológico abordado en 19 estudios $(86,4 \%)$, con los siguientes componentes: deshabituación tabáquica $(n=15)(5,45,46,48,53-$ $55,57,60,62-64,67,71,73)$, práctica regular de ejercicio físico $(n=9)^{(5,46,48,49,54,55,60,63,71)}$, hábitos nutricionales $(n=10){ }_{(5,46-49,53,55,60,62,71)}$, control de las emociones $(n=3)^{(49,55,71)}$, gestión de la disnea $(n=3)^{(49,55,71)}$, técnicas de respiración $(n=6)^{(45,47,55,63,70,71)}$, sueño y 
descanso $(n=4)^{(5,49,53,55)}$, limpieza de secreciones $(n=1)^{(53)}$, sexualidad $(n=1)^{(55)}$, higiene de manos $(n=1)^{(63)}$, pérdida de peso $(n=1)^{(48)}$ y actividades ocio y viajes $(n=1)^{(55)}$.

En relación al tratamiento farmacológico era abortado en el $86,4 \%(n=19)$ de los estudios, específicamente en la instrucción en la técnica de inhalación $(n=12)$ $(5,48,50,54,55,60,63,64,67,70,73,75)$ medicación en las exacerbaciones $(n=7) \quad(5,55,60,63,64,70,71)$, oxigenoterapia $(n=3)^{(47,55,64)}$ y vacunación $(n=3)^{(48,64,73)}$. Haciendo uso del material educativo del Living well with $C O P D^{(76)}$ en 3 estudios.

\section{Medidas de resultado}

Los estudios consultados informaron de las siguientes medidas de resultado:

Variables sociodemográficas (sexo y edad) $(\mathrm{n}=22){ }^{(5,45-50,53-55,62-64,69-71,73-75)}$, calidad de vida relacionada con la salud (CVRS) $(n=17)(5,45-47,49,50,53,55,57,58,60,62,63,67,71,73,74)$, variables ventilatorias: FEV1 $(n=16)^{(5,45,53-55,57,58,60,62,64,67,70,71,73-75)}$, walking Test $(n=5)$ $(55,62,70,73,75)$, FEV1/FVC $(n=9){ }^{(5,53-55,60,64,67,70,71)}$, gasometría arterial $(n=2)^{(5,75)}$, disnea (MRC) $(n=8)^{(5,54,55,60,62,67,70,75)}$, tabaquismo $(n=9)^{(45,46,49,54,60,62,63,67,75)}$, índice de masa corporal (IMC) $(n=5)^{(5,45,46,60,62)}$, comorbilidades $(n=5)^{(5,45,46,60,62)}$, medicación $(n=6)$ $(45,53,55,60,67,73)$, número de ingresos hospitalarios $(n=17)(5,45-50,53,55,57,58,60,62,63,67,75)$, ansiedad y depresión $(n=2)^{(49,75)}$, signos y síntomas $(n=3)^{(5,47,53)}$, satisfacción $(n=3)$ $(53,63,75)$, vacunación $(n=1)^{(47)}$, adherencia terapéutica $(n=1){ }^{(45)}$ y evaluación de la técnica inhalatória $(n=4) \quad(5,58,64,67)$ y el conocimiento de la EPOC $(n=8)$ $(45,48,53,54,60,63,64,71)$

\section{Resultados de los programas educativos}

La intervención educativa disminuye los ingresos hospitalarios y las visitas a urgencias en el $58,8 \%$ de los estudios, favorece o no modifica la deshabituación tabáquica en el 77,77\%, mejora los conocimientos de la EPOC en el $62,5 \%{ }^{(5,45-50,53-}$ 55,62-64,69-71,73-75) y el conocimiento para realizar la técnica de inhalación de forma correcta en el $100 \%$ todos los estudios que así lo han analizado. Así como mejora la satisfacción en el 66,6 \%, como se puede observar en la tabla 1.

El $50 \%$ de los estudios que han evaluado la calidad de vida afirman que no se experimenta un cambio en la misma después de la intervención educativa frente al $43 \%$ que afirma que sí se experimenta una mejora y un único estudio ${ }^{(62)}$ que afirma que perjudica a la intervención educativa. Del mismo modo ocurre en aquellos estudios que evalúan las variables ventilatorias donde el $66,7 \%$ observa que la intervención educativa ni perjudica a las mismas ni afecta negativamente, siendo un $25 \%$ de los estudios que sus resultados muestran que puede beneficiar a los parámetros respiratorios, como se observa en la tabla 1.

Otros parámetros como los signos y los síntomas, en dos ${ }^{(5,53)}$ de los tres estudios que lo analizaron no se vieron afectados ni de forma negativa ni positiva tras la intervención y el restante estudio mostró que era negativo ${ }^{(47)}$. La adherencia farmacológica solo un estudio la han analizado no mejorando, ni disminuyendo la misma tras la intervención educativa ${ }^{(45)}$. Otro de los parámetros estudiados por los investigadores de los distintos ensayos fue el IMC, en uno de ellos solo valorado al inicio de la investigación ${ }^{(46)}$ y aquellos estudios que lo han valorado al inicio y al final han obtenido que mayoritariamente la intervención educativa no mejora este 
parámetro, pero tampoco lo empeora ${ }^{(45,60,62)}$ y solo en uno de ellos ha mejorado tras la intervención educativa ${ }^{(5)}$.

Tabla 1. Efectividad de las principales variables de resultado

\begin{tabular}{|c|c|c|c|c|c|c|c|c|}
\hline & Edad & Sexo & $\begin{array}{l}\text { Seguimiento } \\
\text { (años) }\end{array}$ & $\begin{array}{l}\text { VR } \\
\mathrm{S}\end{array}$ & $\begin{array}{c}\text { V. } \\
\text { ventilatorias }\end{array}$ & $\begin{array}{l}\text { Ingresos, } \\
\text { Visitas hosp }\end{array}$ & Tabaco & $\begin{array}{l}\text { Conocimiento } \\
\text { EPOC }\end{array}$ \\
\hline $\begin{array}{l}\text { Khdour } \\
\text { et al. } 2011\end{array}$ & 6,5 & & 1 & & $=$ & & - & $=$ \\
\hline $\begin{array}{l}\text { Hoogend } \\
\text { oorn et al. } 2010\end{array}$ & 6,5 & & 2 & & & + & & \\
\hline 2009 Koff et al. & 5,8 & & 0 & & & - & & \\
\hline $\begin{array}{l}\text { Siddique } \\
\text { et al. } 2012\end{array}$ & 0,0 & & 1 & & & $=$ & & $=$ \\
\hline al. 2012 Taylor et & 9,8 & & 0 & & & - & & \\
\hline $\begin{array}{l}\text { Bucknall } \\
\text { et al. } 2012\end{array}$ & 9,1 & & 1 & & & 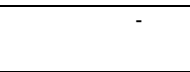 & & \\
\hline al. 2006 Soler et & 3,0 & & 1 & & - & - & & \\
\hline $\begin{array}{l}\text { Watson } \\
\text { et al. } 1997\end{array}$ & 7,5 & & 0 & & $=$ & - & & + \\
\hline $2010 \quad$ Hill et al. & 4,5 & & 0 & & $=$ & & & + \\
\hline $\begin{array}{l}\text { Bourbea } \\
\text { u et al. } 2006\end{array}$ & 9,5 & & 1 & & $=$ & - & & \\
\hline $\begin{array}{l}\text { Coultas } \\
\text { et al. } 2005\end{array}$ & 9,0 & & 0 & & & $=$ & & \\
\hline al. 2012 Kiser et & 3,0 & & 0 & $\begin{array}{l}1 \\
\text { inici } \\
0\end{array}$ & & + & & \\
\hline $\begin{array}{l}\text { Wakabay } \\
\text { ashi et al. } 2011\end{array}$ & 1,7 & & 1 & & $=$ & $=$ & - & + \\
\hline $\begin{array}{l}\text { Theander } \\
\text { et al. } 2009\end{array}$ & 5,0 & & 0 & & $=$ & & & \\
\hline $\begin{array}{l}\text { Efraimss } \\
\text { on et al. } 2008\end{array}$ & 7,0 & & 0 & & + & & - & + \\
\hline $\begin{array}{l}\text { Rootmen } \\
\text { sen et al. } 2008\end{array}$ & 0,5 & & 0 & & & + & & + \\
\hline $\begin{array}{l}\text { Hesselin } \\
\text { k et al. } 2004\end{array}$ & 7,3 & & 2 & & $=$ & & & \\
\hline $2004 \quad$ Rea et al. & 8,0 & & 1 & & + & - & & \\
\hline $\begin{array}{l}\text { Cockcrof } \\
\text { t et al. } 1987\end{array}$ & 9,9 & & & & $=$ & - & - & \\
\hline $\begin{array}{l}\text { Littlejohn } \\
\text { s et al. } 1991\end{array}$ & 2,7 & & 1 & & + & & & \\
\hline al. 2009 Seden et & 9,3 & & 1 & & & & & \\
\hline 2012 Fan et al. & 6,0 & & 1 & & & + & & $=$ \\
\hline $\begin{array}{ll}\text { mujeres. } & \text { * En la variable } \\
& \text { Los valores }+d \\
& \text { Los valores }-d d \\
& \text { Los valores }=d\end{array}$ & $\begin{array}{l}\text { Tas varl } \\
\text { las vari }\end{array}$ & & $\begin{array}{l}\text { a a peores re } \\
\text { ia a resultado }\end{array}$ & & $\begin{array}{l}\text { o intervención } \\
\text { los dos grupos }\end{array}$ & $\begin{array}{l}\text { grupo control } \\
\text { rupo control o } \\
\text { intervención o }\end{array}$ & $\begin{array}{l}\text { especto a } \\
\text { pecto a } \\
\text { pecto a }\end{array}$ & $\begin{array}{l}\text { M hace referencia a las } \\
\text { valuación basal } \\
\text { luación basal } \\
\text { aluación basal }\end{array}$ \\
\hline
\end{tabular}

\section{Calidad metodológica}

En cuanto a la evaluación de la calidad metodológica a través de la escala Jadad, la media total de todos los estudios fue de 3,9. De los 22 estudios incluidos $n=5$ de ellos han obtenido la máxima puntuación metodológica, $n=9$ han obtenido 4 puntos y $n=8$ han obtenido 3 puntos. Siendo el ítem menos valorado la ocultación de la asignación, como consecuencia de la naturaleza de la intervención.

\section{DISCUSIÓN}

La EPOC es una de las enfermedades más prevalentes en los países desarrollados, donde cada vez existe una mayor preocupación por implantar nuevas soluciones a 
estos pacientes, viéndose reflejado en un incremento del número de publicaciones en los últimos 5 años, sobre todo de protocolos de estudio sin que posteriormente se hayan hecho las publicaciones de los resultados de los mismos, acontecimiento también puesto de manifiesto por la revisión Cochrane del año $2011^{(77)}$.

En cuanto al contenido de las sesiones de educación cabe destacar que en esta revisión no se ha hecho una inferencia en los contenidos que pueden afectar positivamente en los mejores resultados de las variables estudiadas, sino que se ha unificado el contenido de los diferentes programas educativos y se ha hecho un descriptivo de los componentes de las mismas. Se han encontrado pocas revisiones que pretendan abordar los componentes de los programas educativos en pacientes con EPOC, donde en uno de ellos, como consecuencia de la heterogeneidad de las intervenciones no se han podido extraer conclusiones de los mismos ${ }^{(77)}$. Pero revisiones como la de Bourbeau, afirman que para obtener beneficios terapéuticos en pacientes con EPOC es necesario elaborar planes individuales, además de dar educación para la salud y un seguimiento telefónico de los mismos ${ }^{(19)}$.

Cabe destacar en los estudios analizados han sido muchos los que han estudiado las variables centradas en el ámbito nutricional, existiendo investigaciones que destacan la importancia de la nutrición en el paciente con EPOC, ya que la dieta rica en hortalizas, frutas y verduras, soja, fibra y aceite de oliva puede favorecer la buena evolución de la enfermedad, frente a dietas ricas en azúcares y carnes curadas que afectan de modo negativo del trascurso de la misma ${ }^{(78)}$, así como la administración de suplementos nutricionales, por el bajo peso corporal que presenta los mismos ya que mejoraría la fuerza de los músculos respiratorios, la actividad física y la calidad de vida $^{(79)}$.

Como se muestra en los resultados obtenidos uno de los componentes que en mayor medida se presenta en los programas educativos en pacientes con EPOC es la deshabituación tabáquica, ya que es el tratamiento que mayor evidencia aporta en la mejora de los pacientes con EPOC, según muestran nuestros resultados siendo efectivos en la deshabituación tabáquica, aunque existen estudios que afirman que la combinación de una intervención psicosocial y un tratamiento farmacológico obtienen mayores tasas de deshabituación, frente a la programas educativos para deshabituación tabáquica ${ }^{(80)}$.

En cuanto a los resultados obtenidos en las variables estudiadas existe un mayor número de estudios que han obtenido una disminución de los ingresos hospitalarios $^{(31)}$, como también ha ocurrido en estudios que han evaluado la calidad de vida en unidades de atención integrada ${ }^{(81)}$ y hospitalización domiciliaria ${ }^{(82)}$ en comparación con la atención convencional. Mayores tasas de deshabituación tabáquica y un aumento de los conocimientos de la EPOC ${ }^{(22)}$, beneficios también reflejados en un estudio llevado a cabo en pacientes como asma y EPOC ${ }^{(25)}$ con respecto a su evaluación basal o el grupo control. Así pues, otras investigaciones también han obtenido resultados positivos en otros aspectos como la calidad de vida $^{(28,29)}$, los parámetros funcionales respiratorios ${ }^{(23,77)}$ y variables que en nuestro caso no se han visto afectadas ni de forma positiva ni negativa. Por el contrario, otras dos revisiones llevadas a cabo, una en el año 2005 y otra en el año 2009, muestran que existe poca evidencia de que los programas educativos en pacientes con EPOC mejoren la calidad de vida, la satisfacción, los reingresos hospitalarios o las visitas a urgencias $^{(13,83)}$. En contra de esos resultados existen programas como la 
teleasistencia aplicada a pacientes con EPOC que han mostrado niveles significativos de mejoría en dichas variables ${ }^{(84)}$.

Otro de los aspectos de gran relevancia en los pacientes crónicos es la adherencia al tratamiento, estudiada en uno de los estudios encontrados, en el que no se mostró ninguna mejoría después de la aplicación de un programa educativo, siendo necesario evaluar la edad, el tratamiento y la patología para mejorar este parámetro $^{(85)}$.

Y programas que incluyen ejercicios de rehabilitación pulmonar y educación sanitaria en EPOC que han obtenido resultados positivos en aspectos como la tolerancia al ejercicio, la disnea y la calidad de vida, siendo más prolongados en el tiempo dicho beneficios comparados con los beneficios de la aplicación de un programa educativo únicamente ${ }^{(30,41,42)}$. No solo siendo beneficiosos para las EPOC, sino también para otras enfermedades crónicas, como pone en evidencia una revisión sistemática del año 2015 que analiza la aplicación de programas de educación terapéutica en paciente con diferentes patologías ${ }^{(86)}$.

En cuanto a la valoración de la calidad metodológica, cabe destacar que nuestros resultados son superiores con los de la revisión elaborada por el equipo de neumología del hospital 12 de Octubre de Madrid ${ }^{(87)}$, donde sus resultados rondan los 2,5 puntos en la escala de Jadad ${ }^{(14)}$. Otra revisión elaborada en el año 2012 en Reino Unido ${ }^{(88)}$ coincide con nuestra investigación en que la calidad metodológica de los estudios localizados es cambiante y los mayores sesgos se presentan en la técnica de ocultación.

\section{Limitaciones metodológicas}

Para intentar disminuir las limitaciones metodológicas se hizo uso de la escala Jadad $^{(14)}$, así como la lista de verificación de estudios PRISMA ${ }^{(89)}$, completando todos los estudios incluidos el $90 \%$ las preguntas estipuladas por la misma. Aspecto que pudo solventarse contactando con los autores de las publicaciones originales, para conocer todos los aspectos desconocidos del estudio. Así como solo se ha utilizado una base de datos para la extracción de información.

\section{CONCLUSIONES}

Según los datos obtenidos desde el año 1987 al año 2013, 22 estudios han analizado la aplicación de la educación terapéutica en pacientes con EPOC, existiendo mayoritariamente más hombres que mujeres, con una media de edad que ronda los 66,4 años.

Las variables más estudiadas fueron la calidad de vida relacionada con la salud, los ingresos hospitalarios, variables ventilatorias como el $\mathrm{FEV}_{1}$, la disnea, el consumo de tabaco, la medicación utilizada y los conocimientos sobre la EPOC. Obteniendo, que en la mayoría de estudios que así lo abordan la educación terapéutica disminuye los ingresos hospitalarios, favorece o no modifica la deshabituación tabáquica, mejora los conocimientos y el manejo de la EPOC, así como la satisfacción con el servicio y los niveles de ansiedad y depresión. No afectando de forma negativa a ninguno de los parámetros estudiados. 
Prácticamente todas las sesiones de educación se llevan a cabo mediante sesiones grupales, apoyadas con folletos informativos, así como los contenidos que en mayor medida se abordan en los programas educativos son descripción de la enfermedad, signos, síntomas, desencadenantes y gestión de una exacerbación, deshabituación tabáquica, práctica regular de ejercicio, hábitos nutricionales e instrucción en la técnica de inhalación.

Cabe destacar de la calidad metodológica, la baja valoración en la ocultación de la asignación como consecuencia de la naturaleza de la intervención.

Coincidiendo con las afirmaciones de todos los estudios analizados es necesaria una mayor investigación en este ámbito, por la incapacidad de poder generar conclusiones que generen grandes evidencias, por la gran variedad de instrumentos utilizados para la evaluación y heterogeneidad de las intervenciones educativas.

\section{REFERENCIAS}

1. Miravitlles M, Soler-Cataluña JJ, Calle M, Molina J, Almagro P, Quintano JA, et al. [Spanish COPD Guidelines (GesEPOC): Pharmacological treatment of stable COPD]. Aten Primaria [Internet]. 2012 Jul [cited 2014 Jun 12];44(7):425-37. Available from: http://www.sciencedirect.com/science/article/pii/S0212656712001850

2. Peces-Barba G, Barberà JA, Agustí A, Casanova C, Casas A, Izquierdo JL, et al. [Diagnosis and management of chronic obstructive pulmonary disease: joint guidelines of the Spanish Society of Pulmonology and Thoracic Surgery (SEPAR) and the Latin American Thoracic Society (ALAT)]. Arch Bronconeumol [Internet]. 2008 May [cited 2014 Jun 12];44(5):271-81. Available from: http://www.ncbi.nlm.nih.gov/pubmed/18448019

3. Anzueto, Antonio; Sethi, Sanjay; Martinez FJ. Exacerbations of chronic obstructive pulmonary disease. Proc Am Thorac Soc [Internet]. 2007 Oct 1 [cited 2014 Jun 8];4(7):554-64. Available from: http://www.ncbi.nlm.nih.gov/pubmed/17878469

4. GESEPOC G de trabajo. Guía de Práctica Clínica para el Diagnóstico y Tratamiento de Pacientes con Enfermedad Pulmonar Obstructiva Crónica (EPOC) [Internet]. [cited 2014 Jun 12]. Available from: file:///C:/Users/Usuario 1/Desktop/6v48nSupl.1a90141716pdf001.pdf

5. Soler JJ, Martínez-García MA, Román P, Orero R, Terrazas S, MartínezPechuán A. [Effectiveness of a specific program for patients with chronic obstructive pulmonary disease and frequent exacerbations]. Arch Bronconeumol [Internet]. 2006 Oct [cited 2014 Jun 11];42(10):501-8. Available from: http://www.ncbi.nlm.nih.gov/pubmed/17067516

6. Miravitlles M, Mayordomo C, Artés M, Sánchez-Agudo L, Nicolau F, Segú JL. Treatment of chronic obstructive pulmonary disease and its exacerbations in general practice. EOLO Group. Estudio Observacional de la Limitación Obstructiva al Flujo aEreo. Respir Med [Internet]. 1999 Mar [cited 2014 Jun 12];93(3):173-9. Available from: http://www.ncbi.nlm.nih.gov/pubmed/10464874

7. Soler J, Sánchez L, Latorre M, Alamar J, Román P, Perpiñá M. [The impact of COPD on hospital resources: the specific burden of COPD patients with high rates of hospitalization]. Arch Bronconeumol [Internet]. 2001 Oct [cited 2014 Jun 12];37(9):375-81. Available from: http://www.ncbi.nlm.nih.gov/pubmed/11674937

8. Zwerink $M$ et al. Self management for patients with chronic obstructive pulmonary disease (Review) [Internet]. Self management for patients with chronic obstructive pulmonary disease (Review). 2014 [cited 2014 Jun 12]. p. Issue 3; The 
Cochrane Collaboration and published. Available from: http://www.updatesoftware.com//BCP/WileyPDF/EN/CD002990.pdf

9. INE. Instituto Nacional de estadística. Sociedad y salud, encuesta de nacional de salud, datos del estado de salud absolutos del año 2012, según problemas o enfermedades crónicas o de larga evolución en los últimos 12 meses en población adulta según sexo y g [Internet]. Encuesta de nacional de salud, datos del estado de salud absolutos del año 2012, según problemas o enfermedades crónicas o de larga evolución en los últimos 12 meses en población adulta. 2012 [cited 2014 Jun 12]. Available from: https://www.google.es/?gws_rd=ssl\#q=.+Instituto+Nacional+de+estad\%C3\%ADstica.+ Sociedad+y+salud\%2C+encuesta+de+nacional+de+salud\%2C+datos+del+estado+de +salud+absolutos+del+a\%C3\%B10+2012\%2C+seg\%C3\%BAn+problemas+o+enferm edades+cr\%C3\%B3nicas+o+de+larga+evoluci\%C3\%B3n+en+los+\%C3\%BAltimos+12 +meses+en+poblaci\%C3\%B3n+adulta+seg\%C3\%BAn+sexo+y+grupo+de+edad.+Pob laci\%C3\%B3n+de+15+y+m\%C3\%A1s+a\%C3\%B1os.+Disponibles+en\%3A+http\%3A \%2F\%2Fwww.ine.es\%2Fjaxi\%2Ftabla.do

10. Global Initiative for Chronic Obstructive Lung Disease 2014 [Internet]. [cited 2014 Jun 12]. Available http://www.goldcopd.org/uploads/users/files/GOLD_Report_2014_Jun11.pdf

11. Steer J, Gibson GJ, Bourke SC. Predicting outcomes following hospitalization for acute exacerbations of COPD. QJM [Internet]. 2010 Nov [cited 2014 Jun 12];103(11):817-29. Available from: http://www.ncbi.nlm.nih.gov/pubmed/20660633

12. Román JM. Interés de la Educación Terapéutica en el manejo del asma. Monografía Asma y Educación. Arch Bronconeumol [Internet]. 2010;46:600-6 V:6573. Available from: http://mail.respirar.org/pdf/gae/curso2006.pdf\#page $=65$

13. Walters JA, Turnock AC, Walters EH, Wood-Baker R. Action plans with limited patient education only for exacerbations of chronic obstructive pulmonary disease. Cochrane database Syst Rev [Internet]. 2010 Jan [cited 2014 Aug 10];(5):CD005074. Available from: http://www.ncbi.nlm.nih.gov/pubmed/20464737

14. Jadad AR, Moore RA, Carroll D, Jenkinson C, Reynolds DJ, Gavaghan DJ, et al. Assessing the quality of reports of randomized clinical trials: is blinding necessary? Control Clin Trials [Internet]. 1996 Mar [cited 2014 May 30];17(1):1-12. Available from: http://www.ncbi.nlm.nih.gov/pubmed/8721797

15. Casey D, Murphy K, Devane D, Cooney A, McCarthy B, Mee L, et al. The effectiveness of a structured education pulmonary rehabilitation programme for improving the health status of people with moderate and severe chronic obstructive pulmonary disease in primary care: the PRINCE cluster randomised trial. Thorax [Internet]. 2013 Oct [cited 2014 May 27];68(10):922-8. Available from: http://www.pubmedcentral. nih.gov/articlerender.fcgi?artid=3786654\&tool=pmcentrez\& $r$ endertype $=$ abstract

16. Parker DR, Eaton CB, Ahern DK, Roberts MB, Rafferty C, Goldman RE, et al. The study design and rationale of the randomized controlled trial: translating COPD guidelines into primary care practice. BMC Fam Pract [Internet]. 2013 Jan [cited 2014 Jun 10];14:56. Available from: http://www.pubmedcentral.nih.gov/articlerender.fcgi?artid=3651367\&tool=pmcentrez\&r endertype=abstract

17. Leiva-Fernández F, Leiva-Fernández J, Zubeldia-Santoyo F, García-Ruiz A, Prados-Torres D, Barnestein-Fonseca P. Efficacy of two educational interventions about inhalation techniques in patients with chronic obstructive pulmonary disease (COPD). TECEPOC: study protocol for a partially randomized controlled trial (preference trial). Trials [Internet]. 2012 Jan [cited 2014 Jun 5];13:64. Available from: 
http://www.pubmedcentral. .nih.gov/articlerender.fcgi?artid=3404981\&tool=pmcentrez\& $r$ endertype $=$ abstract

18. Bourbeau J, Nault D, Dang-Tan T. Self-management and behaviour modification in COPD . Patient Educ Couns [Internet]. 2004 Mar [cited 2014 May 25];52(3):271-7. Available from: http://www.ncbi.nlm.nih.gov/pubmed/14998597

19. Ashmore J, Russo R, Peoples J, Sloan J, Jackson BE, Bae S, et al. Chronic obstructive pulmonary disease self-management activation research trial (COPDSMART): design and methods. Contemp Clin Trials [Internet]. 2013 Jul [cited 2014 Jun 10];35(2):77-86. Available from: http://www.ncbi.nlm.nih.gov/pubmed/23680985

20. Casey D, Murphy K, Cooney A, Mee L. Developing a structured education programme for clients with COPD. Br J Community Nurs [Internet]. 2011 May [cited 2014 Jun 10];16(5):231-7. Available from: http://www.ncbi.nlm.nih.gov/pubmed/21642927

21. Valero C, Monteagudo M, Llagostera M, Bayona X, Granollers S, Acedo M, et al. Evaluation of a combined strategy directed towards health-care professionals and patients with chronic obstructive pulmonary disease (COPD): information and health education feedback for improving clinical monitoring and quality-of-life. BMC Public Health [Internet]. 2009 Jan [cited 2014 May 27];9:442. Available from: http://www.pubmedcentral. .nih.gov/articlerender.fcgi?artid=2799410\&tool=pmcentrez\& $r$ endertype $=$ abstract

22. Paneroni M, Clini E, Crisafulli E, Guffanti E, Fumagalli A, Bernasconi A, et al. Feasibility and effectiveness of an educational program in Italian COPD patients undergoing rehabilitation. Respir Care [Internet]. 2013 Feb [cited 2014 Jun 10];58(2):327-33. Available from: http://www.ncbi.nlm.nih.gov/pubmed/22782190

23. Zhou Y, Hu G, Wang D, Wang S, Wang Y, Liu Z, et al. Community based integrated intervention for prevention and management of chronic obstructive pulmonary disease (COPD) in Guangdong, China: cluster randomised controlled trial. BMJ [Internet]. 2010 Jan [cited 2014 Jun 10];341:c6387. Available from: http://www.pubmedcentral. . nih.gov/articlerender.fcgi?artid=2995286\&tool=pmcentrez\& $r$ endertype $=$ abstract

24. Carré PC, Roche N, Neukirch F, Radeau T, Perez T, Terrioux P, et al. The effect of an information leaflet upon knowledge and awareness of COPD in potential sufferers. A randomized controlled study. Respiration [Internet]. 2008 Jan [cited 2014 Jun 10];76(1):53-60. Available from: http://www.ncbi.nlm.nih.gov/pubmed/18253024

25. Van Zeller M, Vaz AP, Soares Pires F, Neves I, Drummond M, Carvalho AM, et al. Efficacy evaluation of educational sessions for patients with asthma and COPD. Rev Port Pneumol [Internet]. 2012 [cited 2014 Jun 10];18(1):29-33. Available from: http://www.ncbi.nlm.nih.gov/pubmed/22177939

26. Davis AHT, Carrieri-KohIman V, Janson SL, Gold WM, Stulbarg MS. Effects of treatment on two types of self-efficacy in people with chronic obstructive pulmonary disease. J Pain Symptom Manage [Internet]. 2006 Jul [cited 2014 Jun 10];32(1):6070. Available from: http://www.ncbi.nlm.nih.gov/pubmed/16824986

27. Nguyen $\mathrm{HQ}$, Carrieri-Kohlman V. Dyspnea self-management in patients with chronic obstructive pulmonary disease: moderating effects of depressed mood. Psychosomatics [Internet]. 2005 [cited 2014 Jun 10];46(5):402-10. Available from: http://www.ncbi.nlm.nih.gov/pubmed/16145184

28. Finnerty JP, Keeping I, Bullough I, Jones J. The effectiveness of outpatient pulmonary rehabilitation in chronic lung disease: a randomized controlled trial. Chest [Internet]. 2001 Jun [cited 2014 Jun 10];119(6):1705-10. Available from: http://www.ncbi.nlm.nih.gov/pubmed/11399694

29. Kunik ME, Veazey C, Cully JA, Souchek J, Graham DP, Hopko D, et al. COPD education and cognitive behavioral therapy group treatment for clinically significant 
symptoms of depression and anxiety in COPD patients: a randomized controlled trial. Psychol Med [Internet]. 2008 Mar [cited 2014 Jun 10];38(3):385-96. Available from: http://www.ncbi.nlm.nih.gov/pubmed/17922939

30. Scherer YK, Schmieder LE, Shimmel S. The effects of education alone and in combination with pulmonary rehabilitation on self-efficacy in patients with COPD. Rehabil Nurs [Internet]. 1998 [cited 2014 Jun 10];23(2):71-7. Available from: http://www.ncbi.nlm.nih.gov/pubmed/9668868

31. Lainscak M, Kadivec S, Kosnik M, Benedik B, Bratkovic M, Jakhel T, et al. Discharge coordinator intervention prevents hospitalizations in patients with COPD: a randomized controlled trial. J Am Med Dir Assoc [Internet]. 2013 Jun [cited 2014 Jun 10];14(6):450.e1-6. Available from: http://www.ncbi.nlm.nih.gov/pubmed/23623520

32. Gallefoss F. The effects of patient education in COPD in a 1-year follow-up randomised, controlled trial. Patient Educ Couns [Internet]. 2004 Mar [cited 2014 Jun 11];52(3):259-66. Available from: http://www.ncbi.nlm.nih.gov/pubmed/14998595

33. Gallefoss F, Bakke PS. Impact of patient education and self-management on morbidity in asthmatics and patients with chronic obstructive pulmonary disease. Respir Med [Internet]. 2000 Mar [cited 2014 Jun 11];94(3):279-87. Available from: http://www.ncbi.nlm.nih.gov/pubmed/10783940

34. Gallefoss F, Bakke PS. How does patient education and self-management among asthmatics and patients with chronic obstructive pulmonary disease affect medication? Am J Respir Crit Care Med [Internet]. 1999 Dec [cited 2014 Jun 11];160(6):2000-5. Available from: http://www.ncbi.nlm.nih.gov/pubmed/10588620

35. Gallefoss F, Bakke PS, Rsgaard PK. Quality of life assessment after patient education in a randomized controlled study on asthma and chronic obstructive pulmonary disease. Am J Respir Crit Care Med [Internet]. 1999 Mar [cited 2014 Jun 11];159(3):812-7. Available from: http://www.ncbi.nlm.nih.gov/pubmed/10051255

36. Nguyen HQ, Donesky D, Reinke LF, Wolpin S, Chyall L, Benditt JO, et al. Internet-based dyspnea self-management support for patients with chronic obstructive pulmonary disease. J Pain Symptom Manage [Internet]. 2013 Jul [cited 2014 Jun 11];46(1):43-55. Available from: http://www.ncbi.nlm.nih.gov/pubmed/23073395

37. Lord VM, Cave P, Hume VJ, Flude EJ, Evans A, Kelly JL, et al. Singing teaching as a therapy for chronic respiratory disease--a randomised controlled trial and qualitative evaluation. BMC Pulm Med [Internet]. 2010 Jan [cited 2014 Jun 11];10:41. Available from: http://www.pubmedcentral.nih.gov/articlerender.fcgi?artid=2920262\&tool=pmcentrez\&r endertype $=$ abstract

38. Osterlund Efraimsson E, Osterlund Eraimsson E, Eva OE, Klang B, Birgitta K, Larsson $\mathrm{K}$, et al. Communication and self-management education at nurse-led COPD clinics in primary health care. Patient Educ Couns [Internet]. 2009 Nov [cited 2014 Jun 11];77(2):209-17. Available from: http://www.ncbi.nlm.nih.gov/pubmed/19414234

39. Kim J, Kim S, Kim H-C, Kim K-H, Yang S-C, Lee C-T, et al. Effects of consumer-centered u-health service for the knowledge, skill, and attitude of the patients with chronic obstructive pulmonary disease. Comput Inform Nurs [Internet]. 2012 Dec [cited 2014 Jun 10];30(12):661-71. Available from: http://www.ncbi.nlm.nih.gov/pubmed/23266537

40. Kara M, Aşti T. Effect of education on self-efficacy of Turkish patients with chronic obstructive pulmonary disease. Patient Educ Couns [Internet]. 2004 Oct [cited 2014 Jun 11];55(1):114-20. Available from: http://www.ncbi.nlm.nih.gov/pubmed/15476998

41. Boxall A-M, Barclay L, Sayers A, Caplan GA. Managing chronic obstructive pulmonary disease in the community. A randomized controlled trial of home-based pulmonary rehabilitation for elderly housebound patients. $J$ Cardiopulm Rehabil 
[Internet]. 2005 [cited 2014 Jun 10];25(6):378-85. Available from: http://www.ncbi.nlm.nih.gov/pubmed/16327534

42. Carrieri-Kohlman V, Nguyen HQ, Donesky-Cuenco D, Demir-Deviren S, Neuhaus J, Stulbarg MS. Impact of brief or extended exercise training on the benefit of a dyspnea self-management program in COPD. J Cardiopulm Rehabil [Internet]. 2005 [cited $2014 \quad$ May 29];25(5):275-84. Available from: http://www.ncbi.nlm.nih.gov/pubmed/16217231

43. McGlone S, Wood-Baker R WE. The effect of a written action plan in COPD [Abstract]. Respirology 2004;9(2 Suppl):A46. Respirology [Internet]. 2004 Mar [cited 2014 Sep 23];9(s2):A1-A75. Available from: http://doi.wiley.com/10.1111/j.14401843.2004.00553.x

44. Field SK, Conley DP, Thawer AM, Leigh R, Cowie RL. Effect of the management of patients with chronic cough by pulmonologists and certified respiratory educators on quality of life: a randomized trial. Chest [Internet]. 2009 Oct [cited 2014 Jun 11];136(4):1021-8. Available from: http://www.ncbi.nlm.nih.gov/pubmed/19349387 45. Khdour MR, Agus AM, Kidney JC, Smyth BM, McElnay JC, Elnay JC, et al. Cost-utility analysis of a pharmacy-led self-management programme for patients with COPD. Int J Clin Pharm [Internet]. 2011 Aug [cited 2014 Jun 11];33(4):665-73. Available from: http://www.ncbi.nlm.nih.gov/pubmed/21643784

46. Hoogendoorn $M$, van Wetering CR, Schols AM, Rutten-van Mölken MPMH. Is INTERdisciplinary COMmunity-based COPD management (INTERCOM) costeffective? Eur Respir J [Internet]. 2010 Jan [cited 2014 Jun 11];35(1):79-87. Available from: http://www.ncbi.nlm.nih.gov/pubmed/19574331

47. Koff PB, Jones RH, Cashman JM, Voelkel NF, Vandivier RW. Proactive integrated care improves quality of life in patients with COPD. Eur Respir $\mathrm{J}$ [Internet]. 2009 May [cited 2014 Jun 11];33(5):1031-8. Available from: http://www.ncbi.nlm.nih.gov/pubmed/19129289

48. Siddique HH, Olson RH, Parenti CM, Rector TS, Caldwell M, Dewan NA, et al. Randomized trial of pragmatic education for low-risk COPD patients: impact on hospitalizations and emergency department visits. Int $\mathrm{J}$ Chron Obstruct Pulmon Dis [Internet]. 2012 Jan [cited 2014 Jun 11];7:719-28. Available from: http://www.pubmedcentral.nih.gov/articlerender.fcgi?artid=3484530\&tool=pmcentrez\&r endertype $=$ abstract

49. Taylor SJC, Sohanpal R, Bremner SA, Devine A, McDaid D, Fernández J-L, et al. Self-management support for moderate-to-severe chronic obstructive pulmonary disease: a pilot randomised controlled trial. $\mathrm{Br} \mathrm{J}$ Gen Pract [Internet]. 2012 Oct [cited 2014 Jun 11];62(603):e687-95. Available from: http://www.pubmedcentral. .nih.gov/articlerender.fcgi?artid=3459776\&tool=pmcentrez\& $r$ endertype $=$ abstract

50. Bucknall CE, Miller G, Lloyd SM, Cleland J, McCluskey S, Cotton M, et al. Glasgow supported self-management trial (GSuST) for patients with moderate to severe COPD: randomised controlled trial. BMJ [Internet]. 2012 Jan [cited 2014 Jun 11];344:e1060. Available from: http://www.pubmedcentral.nih.gov/articlerender.fcgi?artid=3295724\&tool=pmcentrez\&r endertype=abstract

51. Chavannes NH, Grijsen M, van den Akker M, Schepers H, Nijdam M, Tiep B, et al. Integrated disease management improves one-year quality of life in primary care COPD patients: a controlled clinical trial. Prim Care Respir J [Internet]. 2009 Sep [cited 2014 Jun 11];18(3):171-6. Available from: http://www.ncbi.nlm.nih.gov/pubmed/19142557

52. Gallefoss F, Bakke PS. Patient satisfaction with healthcare in asthmatics and patients with COPD before and after patient education. Respir Med [Internet]. 2000 
Nov [cited 2014 Jun 3];94(11):1057-64. Available from: http://www.ncbi.nlm.nih.gov/pubmed/11127492

53. Watson PB, Town Gl, Holbrook N, Dwan C, Toop LJ, Drennan CJ. Evaluation of a self-management plan for chronic obstructive pulmonary disease. Eur Respir $\mathrm{J}$ [Internet]. 1997 Jun [cited 2014 Jun 11];10(6):1267-71. Available from: http://www.ncbi.nlm.nih.gov/pubmed/9192927

54. Hill K, Mangovski-Alzamora S, Blouin M, Guyatt G, Heels-Ansdell D, Bragaglia $P$, et al. Disease-specific education in the primary care setting increases the knowledge of people with chronic obstructive pulmonary disease: a randomized controlled trial. Patient Educ Couns [Internet]. 2010 Oct [cited 2014 Jun 11];81(1):148. Available from: http://www.ncbi.nlm.nih.gov/pubmed/19853399

55. Bourbeau J, Collet J-P, Schwartzman K, Ducruet T, Nault D, Bradley C. Economic benefits of self-management education in COPD. Chest [Internet]. 2006 Dec [cited 2014 Jun 11];130(6):1704-11. Available from: http://www.ncbi.nlm.nih.gov/pubmed/17166985

56. Monninkhof $E$, van der Valk $P$, Schermer $T$, van der Palen J, van Herwaarden $\mathrm{C}$, Zielhuis $\mathrm{G}$. Economic evaluation of a comprehensive self-management programme in patients with moderate to severe chronic obstructive pulmonary disease. Chron Respir Dis [Internet]. 2004 Jan [cited 2014 Jun 11];1(1):7-16. Available from: http://www.ncbi.nlm.nih.gov/pubmed/16281663

57. Coultas D, Frederick J, Barnett B, Singh G, Wludyka P. A randomized trial of two types of nurse-assisted home care for patients with COPD. Chest [Internet]. 2005 Oct [cited 2014 Jun 11];128(4):2017-24. Available from: http://www.ncbi.nlm.nih.gov/pubmed/16236850

58. Kiser K, Jonas D, Warner Z, Scanlon K, Shilliday BB, DeWalt DA. A randomized controlled trial of a literacy-sensitive self-management intervention for chronic obstructive pulmonary disease patients. J Gen Intern Med [Internet]. 2012 Feb [cited 2014 Jun 11];27(2):190-5. Available from: http://www.pubmedcentral. .nih.gov/articlerender.fcgi?artid=3270237\&tool=pmcentrez\& $r$ endertype $=$ abstract

59. Monteagudo M, Rodriguez-Blanco T, Llagostera M, Valero C, Bayona X, Granollers S, et al. Effect of health professional education on outcomes of chronic obstructive pulmonary disease in primary care: a non-randomized clinical trial. Respirology [Internet]. 2013 May [cited 2014 Jun 11];18(4):718-27. Available from: http://www.ncbi.nlm.nih.gov/pubmed/23438203

60. Wakabayashi R, Motegi T, Yamada K, Ishii T, Jones RC, Hyland ME, et al. Efficient integrated education for older patients with chronic obstructive pulmonary disease using the Lung Information Needs Questionnaire. Geriatr Gerontol Int [Internet]. 2011 Oct [cited 2014 Jun 11];11(4):422-30. Available from: http://www.ncbi.nlm.nih.gov/pubmed/21447136

61. Rice KL, Dewan N, Bloomfield HE, Grill J, Schult TM, Nelson DB, et al. Disease management program for chronic obstructive pulmonary disease: a randomized controlled trial. Am J Respir Crit Care Med [Internet]. 2010 Oct 1 [cited 2014 Jun 11];182(7):890-6. Available from: http://www.ncbi.nlm.nih.gov/pubmed/20075385

62. Theander K, Jakobsson $P$, Jörgensen N, Unosson M. Effects of pulmonary rehabilitation on fatigue, functional status and health perceptions in patients with chronic obstructive pulmonary disease: a randomized controlled trial. Clin Rehabil [Internet]. 2009 Feb [cited 2014 Jun 11];23(2):125-36. Available from: http://www.ncbi.nlm.nih.gov/pubmed/19164400

63. Efraimsson EO, Hillervik C, Ehrenberg A. Effects of COPD self-care management education at a nurse-led primary health care clinic. Scand J Caring Sci 
[Internet]. 2008 Jun [cited 2014 Jun 11];22(2):178-85. Available from: http://www.ncbi.nlm.nih.gov/pubmed/18489687

64. Rootmensen GN, van Keimpema ARJ, Looysen EE, van der Schaaf L, de Haan $\mathrm{RJ}$, Jansen HM. The effects of additional care by a pulmonary nurse for asthma and COPD patients at a respiratory outpatient clinic: results from a double blind, randomized clinical trial. Patient Educ Couns [Internet]. 2008 Feb [cited 2014 Jun 11];70(2):179-86. Available from: http://www.ncbi.nlm.nih.gov/pubmed/18031971

65. Sridhar M, Taylor R, Dawson S, Roberts NJ, Partridge MR. A nurse led intermediate care package in patients who have been hospitalised with an acute exacerbation of chronic obstructive pulmonary disease. Thorax [Internet]. 2008 Mar [cited 2014 Jun 11];63(3):194-200. Available from: http://www.ncbi.nlm.nih.gov/pubmed/17901162

66. Gadoury M-A, Schwartzman K, Rouleau M, Maltais F, Julien M, Beaupré A, et al. Self-management reduces both short- and long-term hospitalisation in COPD. Eur Respir J [Internet]. 2005 Nov [cited 2014 Jun 11];26(5):853-7. Available from: http://www.ncbi.nlm.nih.gov/pubmed/16264046

67. Hesselink AE, Penninx BWJH, van der Windt DAWM, van Duin BJ, de Vries $P$, Twisk JWR, et al. Effectiveness of an education programme by a general practice assistant for asthma and COPD patients: results from a randomised controlled trial. Patient Educ Couns [Internet]. 2004 Oct [cited 2014 Jun 11];55(1):121-8. Available from: http://www.ncbi.nlm.nih.gov/pubmed/15476999

68. Bischoff EWMA, Akkermans R, Bourbeau J, van Weel C, Vercoulen JH, Schermer TRJ. Comprehensive self management and routine monitoring in chronic obstructive pulmonary disease patients in general practice: randomised controlled trial. BMJ [Internet]. 2012 Jan 28 [cited 2014 Jun 24];345(nov28_1):e7642. Available from: http://www.bmj.com/content/345/bmj.e7642.full.pdf+html

69. McGeoch GRB, Willsman KJ, Dowson CA, Town GI, Frampton CM, McCartin FJ, et al. Self-management plans in the primary care of patients with chronic obstructive pulmonary disease. Respirology [Internet]. 2006 Sep [cited 2014 Jun 10];11(5):611-8. Available from: http://www.ncbi.nlm.nih.gov/pubmed/16916335

70. Sedeno MF, Nault D, Hamd DH, Bourbeau J. A self-management education program including an action plan for acute COPD exacerbations. COPD [Internet]. 2009 Oct [cited 2014 Jun 10];6(5):352-8. Available from: http://www.ncbi.nlm.nih.gov/pubmed/19863364

71. Fan VS, Gaziano JM, Lew R, Bourbeau J, Adams SG, Leatherman S, et al. A comprehensive care management program to prevent chronic obstructive pulmonary disease hospitalizations: a randomized, controlled trial. Ann Intern Med [Internet]. 2012 May 15 [cited 2014 May 23];156(10):673-83. Available from: http://www.ncbi.nlm.nih.gov/pubmed/22586006

72. Labrecque M, Rabhi K, Laurin C, Favreau H, Moullec G, Lavoie K, et al. Can a self-management education program for patients with chronic obstructive pulmonary disease improve quality of life? Can Respir J [Internet]. 2011 [cited 2014 Jun 11];18(5):e77-81. Available from: http://www.pubmedcentral.nih.gov/articlerender.fcgi?artid=3267612\&tool=pmcentrez\&r endertype $=$ abstract

73. Rea H, McAuley S, Stewart A, Lamont C, Roseman P, Didsbury P. A chronic disease management programme can reduce days in hospital for patients with chronic obstructive pulmonary disease. Intern Med J [Internet]. 2004 Nov [cited 2014 Jun 11];34(11):608-14. Available from: http://www.ncbi.nlm.nih.gov/pubmed/15546454

74. Cockcroft A, Bagnall P, Heslop A, Andersson N, Heaton R, Batstone J, et al. Controlled trial of respiratory health worker visiting patients with chronic respiratory disability. $\mathrm{Br}$ Med J (Clin Res Ed) [Internet]. 1987 Jan 24 [cited 2014 Jun 
http://www.pubmedcentral.nih.gov/articlerender.fcgi?artid=1245234\&tool=pmcentrez\&r endertype $=$ abstract

75. Littlejohns P, Baveystock CM, Parnell H, Jones PW. Randomised controlled trial of the effectiveness of a respiratory health worker in reducing impairment, disability, and handicap due to chronic airflow limitation. Thorax [Internet]. 1991 Aug [cited 2014 Jun 11];46(8):559-64. Available from: http://www.pubmedcentral. . nih.gov/articlerender.fcgi?artid=463273\&tool=pmcentrez\&re ndertype $=$ abstract

76. Living well with a Chronic Obstructive Pulmonary Disease - COPD [Internet]. [cited 2014 Nov 19]. Available from: http://www.livingwellwithcopd.com/

77. Cochrane Data Base Of Systematic Reviews. Autocuidado para pacientes con enfermedad obstructiva crónica (Revisión Cochrane traducida). [Internet]. 2011 [cited 2014 Jul 16]. Available from: http://www.update-software.com/PDF-ES/CD002990.pdf

78. De los Santos Izquierdo JM, Peña Molinero C, Ortega Barriuso R, Manso Melgosa AB. Revisión de evidencias sobre la influencia de la dieta en la evolución de la EPOC. Enfermería Glob [Internet]. 2012 Oct 1 [cited 2014 Jul 16];11(4). Available from: http://revistas.um.es/eglobal/article/view/eglobal.11.4.132491

79. Ferreira IM, Brooks D, White J, Goldstein R. Nutritional supplementation for stable chronic obstructive pulmonary disease. Cochrane database Syst Rev [Internet]. 2012 Jan [cited 2014 Aug 25];12:CD000998. Available from: http://www.ncbi.nlm.nih.gov/pubmed/23235577

80. Van der Meer RM, Wagena EJ, Ostelo RWJG, Jacobs JE, van Schayck CP. Smoking cessation for chronic obstructive pulmonary disease. Cochrane database Syst Rev [Internet]. 2003 Jan [cited 2014 Aug 25];(2):CD002999. Available from: http://www.ncbi.nlm.nih.gov/pubmed/12804448

81. Casas A, Troosters T, Garcia-Aymerich J, Roca J, Hernández C, Alonso A, et al. Integrated care prevents hospitalisations for exacerbations in COPD patients. Eur Respir J [Internet]. 2006 Jul 1 [cited 2015 Jul 20];28(1):123-30. Available from: http://erj.ersjournals.com/content/28/1/123.full

82. Hernandez C, Casas A, Escarrabill J, Alonso J, Puig-Junoy J, Farrero E, et al. Home hospitalisation of exacerbated chronic obstructive pulmonary disease patients. Eur Respir J [Internet]. 2003 Jan [cited 2014 Jun 16];21(1):58-67. Available from: http://www.ncbi.nlm.nih.gov/pubmed/12570110

83. Taylor SJC, Candy B, Bryar RM, Ramsay J, Vrijhoef HJM, Esmond G, et al. Effectiveness of innovations in nurse led chronic disease management for patients with chronic obstructive pulmonary disease: systematic review of evidence. BMJ [Internet]. 2005 Sep 3 [cited 2014 Jul 17];331(7515):485. Available from: http://www.bmj.com/content/331/7515/485

84. Rühle K-H. [COPD: Living better with telehealthcare?]. Dtsch Med Wochenschr [Internet]. 2012 Apr [cited 2014 Aug 10];137(16):827. Available from: http://www.ncbi.nlm.nih.gov/pubmed/22495916

85. Jansà $M$, Hernández $C$, Vidal $M$, Nuñez $M$, Bertran $M J$, Sanz $S$, et al. Multidimensional analysis of treatment adherence in patients with multiple chronic conditions. A cross-sectional study in a tertiary hospital. Patient Educ Couns [Internet]. Elsevier; 2010 Nov 11 [cited 2015 Jul 20];81(2):161-8. Available from: http://www.pecjournal.com/article/S0738399110000078/fulltext

86. Paul Galdas; Jennifer Fell; Peter Bower; Lisa Kidd. The effectiveness of selfmanagement support interventions for men with long-term conditions: a systematic review and meta-analysis [Internet]. 2014 [cited 2015 Mar 21]. Available from: file://C:/Users/Usuario 1/Downloads/BMJ Open-2015-Galdas-.pdf 
87. Octubre $\mathrm{G}$ de $\mathrm{MB}$ en la EHU 12 de O. EFICACIA DE LOS PROGRAMAS DE TRATAMIENTO INTEGRAL DE LA EPOC. REVISIÓN SISTEMÁTICA Y METAANÁLISIS DE ARTÍCULOS [Internet]. Evidence Based Medicine Neumología. 2014 [cited 2014 Jul 16]. Available from: http://mbeneumologia.org/mbe/docsMBE/cat.aspx?sec=tra\&id=87\&p=e

88. Sohanpal R, Hooper R, Hames R, Priebe S, Taylor S. Reporting participation rates in studies of non-pharmacological interventions for patients with chronic obstructive pulmonary disease: a systematic review. Syst Rev [Internet]. BioMed Central Ltd; 2012 Jan 29 [cited 2014 Jul 16];1(1):66. Available from: http://www.systematicreviewsjournal.com/content/1/1/66

89. Shamseer L, Moher D, Clarke M, Ghersi D, Liberati A, Petticrew $M$, et al. Preferred reporting items for systematic review and meta-analysis protocols (PRISMAP) 2015: elaboration and explanation. BMJ [Internet]. 2015 Jan [cited 2015 Feb 10];349:g7647. Available from: http://www.ncbi.nlm.nih.gov/pubmed/25555855

Recibido: 27 de enero 2016;

Aceptado: 15 de abril 2016

ISSN 1695-6141

() COPYRIGHT Servicio de Publicaciones - Universidad de Murcia 